\title{
Correlation between Joint Ulttrasound Data and Clinical, Laboratory and Instrumental Indices in Patients with Rheumatoid Arthritis
}

\author{
DOI: 10.17691/stm2017.9.1.11
}

Received September 21, 2015

E,B. Komarova, MD, PhD, Associate Professor, Internal Medicine Department,

Faculty of Postgraduate Studies

Lugansk State Medical University, 1g, 50 let Oborony St., Lugansk, 91031

The aim of the investigation was to assess the correlation between the main ultrasound indices of joints and clinical, laboratory and instrumental indices in patients with rheumatoid arthritis (RA).

Materials and Methods. A total of 114 patients with RA underwent complex clinical, laboratory and instrumental examination, including Clinical assessment of the patients' health, according to RA activity index DAS28. Concentrations of C-reactive protein (CRP), TNF- $\alpha$, anti-cyclic citrullinated peptide antibodies (ACPA), angiogenesis marker VEGF and proliferation factor FGF were measured using ELISA technique. Ultrasound examination of knee joints was carried out using ESAOTE MyLAB40 device (The Netherlands), the knee joint arthroscopy was performed using an arthroscope (Karl Storz, Germany) with a diameter of $2.4 \mathrm{~mm}$ and an angle of $30^{\circ}$. The synovial membrane samples were fixed in $10 \%$ buffered formalin solution, stained with hematoxylin and eosin and studied using Axiostar microscope (Carl Zeiss, Germany).

Results. There were established statistically significant correlations between the deterioration of ultrasound indices of synovitis, synovial membrane vascularization and the increase in the markers of immune-inflammatory RA activity and VEGF marker in the blood, which indicated decline in clinical assessment of the patients' health. The increase in ultrasound indices of synovial membrane thickness, pannus, osteochondral erosions closely correlated with the increase in RA duration, high blood levels of CRP, ACPA and FGF, which also spoke of deterioration of the clinical indices of the disease and radiographic progression of joint destruction.

Conclusion. The intensity of immune-inflammatory, proliferative and destructive processes and the synovial membrane angiogenesis can be assessed by ultrasound examination of joints in RA patients. The data obtained provide the possibility of recommending the application of the non-invasive technique of synovial membrane ultrasound for diagnosis and monitoring treatment efficacy in RA patients instead of expensive invasive techniques.

Key words: rheumatoid arthritis; synovial membrane; rheumatoid arthritis activity; arthroscopy.

Rheumatoid arthritis (RA) is a chronic autoimmune inflammatory destructive joint disease characterized by damage to the synovial membrane (SM) as the main target organ [1]. Angiogenesis and fibroblast proliferation are important elements of the multilevel pathogenesis of SM hyperplasia development in RA [2]. Tissue hypoxia, pro-inflammatory cytokines and growth factors, such as vascular endothelial growth factor (VEGF) and fibroblast growth factor (FGF), promote angiogenesis processes which, in their turn, increase infiltration and SM hyperplasia, pannus mass growth and osteochondral erosion development [3].

Various instrumental methods for joint structure examination are used to diagnose RA: radiological examination, computerized and magnetic resonance tomography, ultrasound imaging, arthroscopy. Arthroscopy can be applied with both diagnostic (macroassessment of the SM with biopsy followed by histologic examination) and therapeutic purposes $[4,5]$.

Ultrasound imaging of joints has numerous advantages as compared to radiological examination, since it provides the possibility to reveal inflammatory and destructive changes in periarticular soft tissues even early in the course of RA, to detect the presence of the minimum amount of free fluid in the joint cavity and periarticular capsules and to evaluate the character of the fluid. Ultrasound imaging detects changes in SM and pannus formation early in the course of RA development, evaluates their vascularization using Doppler scanning and also helps reveal osteochondral erosions when no destruction is found by X-ray examination [68]. Ultrasound imaging of joints is a highly reliable informative non-invasive technique (unlike arthroscopy), it is cost-efficient and affordable (compared to computed tomography and magnetic reconance imaging), enables making repeated dynamic examinations in the course of conducted therapy, has no contraindications [9].

A number of studies aiming to evaluate the ratio of indices provided by ultrasound of joints and clinical and laboratory parameters in RA report different results: some

For contacts: Elena B. Komarova, e-mail: elbelcom@ua.fm 
authors have established positive correlation with the patients' health assessment indices (HAQ scale index, the Ritchie Index and RA activity index DAS28), but have found no correlation with erythrocyte sedimentation rate $(E S R)$ and $C$-reactive protein (CRP) parameters [10]; others have found no correlation with DAS28 index improvement and decrease in SM vascularization intensity in RA patients after treatment [11].

Such situation determines the need to study interrelations between cross-links of pathogenesis using the data of profound analysis and comparison of joint ultrasound imaging and clinical laboratory indices in RA patients.

The aim of the investigation was to assess the correlation between the main ultrasound indices of joints and clinical, laboratory and instrumental indices in patients with rheumatoid arthritis.

Materials and Methods. There were examined 114 patients with RA (the diagnosis verified according to ACR/EULAR criteria, 2010) without concomitant pathology. Females comprised $86.6 \%$ of patients, males were $13.4 \%$. The patients' age amounted to $22-65$ years (the mean age was $47.70 \pm 10.22$ years), the mean disease duration was $3.82 \pm 3.43$ years. The radiologic stage was determined according to the Steinbrocker criteria: stage 0 (absence of changes) was established in 18 patients $(15.9 \%)$, stage I in $42(36.6 \%)$, stage II in 22 (19.6\%), stage III in $19(17.1 \%)$ and stage IV was found in 13 patients (10.8\%). The following parameters were clinically evaluated: joint counts (the tender joint count (TJC), the swollen joint count (SJC)), the patients' health according to $\mathrm{HAQ}$ scale, assessment of pain intensity using visual analogue scale (VAS). RA activity was assessed according to index DAS28, with the formula containing the value of CRP in patients' blood. Serum concentrations of CRP and TNF- $\alpha$ (Vector-Best, Russia), anti-citrullinated protein antibodies (ACPA) (Euroimmun, Germany), VEGF and FGF (BCM Diagnostic, Canada) were measured using ELISA technique.

Ultrasound imaging of the knee joints was performed using ESAOTE MyLAB40 device (Esaote, The Netherlands) with a linear array probe $7.5 \mathrm{~L} 70$ (frequency $7.5 \mathrm{MHz}$ ). The following parameters were evaluated: exudation into the articular cavity, SM thickness and vascularization, the presence of pannus and osteochondral erosions. The knee joint arthroscopy with macro-assessment of the SM was performed in 33 patients (for chronic recurrent synovitis refractory to basic therapy) using Karl Storz arthroscope (Germany) with a diameter of $2.4 \mathrm{~mm}$ and an angle of $30^{\circ}$.

Macro-assessment involved the indices of inflammatory and synovial villous hyperplasia, hyperemia with increased vascular pattern, identifying the presence of pannus and fibrin. SM samples were obtained using biopsy forceps with a diameter of $3.5 \mathrm{~mm}$ (Karl Storz, Germany), fixed in $10 \%$ buffered formalin solution ( $\mathrm{pH} 7.0)$, stained with hematoxylin and eosin. The preparations were studied in transmitted light using Axiostar microscope (Carl Zeiss, Germany). SM micro-assessment involved revealing SM edema, hyperplasia of synovial villi with tectorial cell proliferation, angiogenesis signs, lymphoid infiltration, connective tissue disorganization. Semi-quantitative assessment was used [4].

The study complies with the Declaration of Helsinki (adopted in June 1964, Helsinki, Finland and revised in October 2000, Edinburgh, Scotland) and was performed following approval by the Ethic Committee of Lugansk State Medical University. Written informed consent was obtained from every patient under study.

The obtained data were statistically processed using non-parametric methods, correlational multivariate (ANOVA/MANOVA) analysis of variance and license software Microsoft Excel and Statistica (StatSoft, USA). There were assessed Spearman correlation coefficient $(R)$, Fisher's criterion $(F)$, reliability of statistic indices (p). Statistically significant differences were determined at the level of $p<0.05$.

Results. There were found positive correlations between ultrasound index of exudation into the articular cavity and the indices of SJC $(R=0.31 ; p=0.0038)$, VAS $(R=0.34 ; p=0.00056), E S R(R=0.37 ; p=0.00036), D A S 28$ $(R=0.35 ; p=0.00042)$, TNF- $\alpha(R=0.34 ; p=0.0006)$ and VEGF ( $R=0.42 ; p=0.00009)$, but there was no correlation with sex and age of the patients, RA duration, radiologic stage, TJC, HAQ scale, the blood levels of ACPA, FGF. This ultrasound index also positively correlated with arthroscopic indices of SM macro-assessment: inflammatory hyperplasia $(R=0.35 ; \quad p=0.025), \quad S M$ hyperemia $(R=0.31 ; p=0.042)$ and fibrin presence $(R=0.39 ; p=0.007)$. There were also found positive correlations with morphological indicators of SM edema and angiogenesis $(R=0.41 ; p=0.0032$ and $R=0.31$; $p=0.042$, respectively).

There were revealed weak positive correlations between ultrasound index of SM thickness and TNF- $\alpha$ index $(R=0.23 ; p=0.031)$, moderate positive correlations with VAS $(R=0.25 ; p=0.01)$, strong correlations with $\mathrm{RA}$ duration $(\mathrm{R}=0.44 ; \mathrm{p}=0.0012)$, the radiologic stage $(R=0.52 ; p<0.001)$ and the blood level of FGF $(R=0.49$; $p=0.00019)$. There were established positive correlations between this index and the arthroscopic indices of villous hyperplasia and pannus presence $(R=0.41 ; p=0.01$ and $R=0.51 ; p=0.00017$, respectively). There were also found positive correlations between SM thickness and morphological indicators of villous hyperplasia with tectorial cell proliferation $(R=0.46 ; p=0.0034)$. Notably, there was observed a tendency towards positive correlation with the indicator of lymphoid SM infiltration, though it was not statistically significant $(R=0.29$; $\mathrm{p}=0.05)$.

Ultrasound index of SM vascularization had strong positive correlations with VAS $(R=0.32 ; p=0.0014)$, TNF- $\alpha \quad(R=0.35 ; \quad p=0.00016)$ and VEGF $(R=0.61$; $p<0.001)$, moderate positive correlations with $H A Q$ scale $(R=0.29 ; p=0.01)$, ESR $(R=0.29 ; p=0.01)$ and $D A S 28$ 
Table 1

The results of multivariate ANOVA/MANOVA analysis of the influence of joint ultrasound indices on rheumatoid arthritis activity index DAS28

\begin{tabular}{lccccc}
\hline \multicolumn{1}{c}{ Indices } & SS & df & MS & F criterion & $p$ \\
\hline Exudation into the articular cavity & 12.1 & 2 & 6.07 & 8.99 & 0.0002 \\
\hline Synovial membrane thickness & 0.49 & 3 & 0.16 & 0.21 & 0.89 \\
Synovial membrane vascularization & 8.99 & 3 & 2.99 & 4.23 & 0.007 \\
\hline Pannus & 1.78 & 3 & 0.594 & 0.767 & 0.52 \\
Erosions & 4.13 & 3 & 1.375 & 1.824 & 0.15 \\
\hline
\end{tabular}

N o t e. SS: the sum of squared deviations; df: the number of degrees of freedom; MS: assessment of variance between sample data.

Table 2

The results of multivariate ANOVA/MANOVA analysis of the influence of joint ultrasound indices on the radiologic stage of rheumatoid arthritis

\begin{tabular}{lccccc}
\hline \multicolumn{1}{c}{ Indices } & SS & df & MS & F criterion & p \\
\hline Exudation into the articular cavity & 3.47 & 2 & 1.73 & 1.73 & 0.18 \\
\hline Synovial membrane thickness & 39.31 & 3 & 13.10 & 19.19 & $<0.001$ \\
Synovial membrane vascularization & 12.33 & 3 & 4.11 & 4.42 & 0.006 \\
\hline Pannus & 76.49 & 3 & 25.49 & 74.62 & $<0.001$ \\
\hline Erosions & 77.77 & 3 & 25.92 & 78.56 & $<0.001$ \\
\hline
\end{tabular}

$\mathrm{N}$ o t e. SS: the sum of squared deviations; df: the number of degrees of freedom; MS: assessment of variance between sample data.

$(R=0.29 ; p=0.01)$, weak correlations with $C R P(R=0.21$; $p=0.042)$ and ACPA $(R=0.22 ; p=0.036)$. There were also found negative correlations between this index and RA duration $(R=-0.39 ; p=0.0019)$ and FGF level $(R=-0.23$; $p=0.035)$. Analysis of correlations between utrasound index of SM vascularization and SM macro-assessment indices revealed strong positive correlations with SM hyperemia $(R=0.61 ; p<0.001)$ and pannus presence $(R=0.31 ; p=0.025)$. There were also established positive correlations with morphological indicators of $\mathrm{SM}$ angiogenesis and lymphoid infiltration $(R=0.55$; $p=0.00011$ and $R=0.30 ; p=0.037$, respectively).

Ultrasound index of pannus formation had strong positive correlations with $R A$ duration $(R=0.71 ; p<0.001)$, the radiologic stage $(R=0.66 ; p<0.001)$ and the blood level of FGF $(R=0.58 ; p=0.00001)$, weak correlations with TJC $(R=0.26 ; p=0.037)$. Analysis of correlations between ultrasound index of pannus and arthroscopic SM indices revealed positive correlations with the indices of villous hyperplasia, hyperemia and pannus presence $(R=0.36 ; p=0.02 ; R=0.31 ; p=0.03$ and $R=0.61$; $p<0.001$, respectively). There were also established positive correlations with morphological indicators of villous hyperplasia with tectorial cell proliferation and angiogenesis $(R=0.46 ; p=0.01$ and $R=0.31 ; p=0.03$, respectively).

Ultrasound index of osteochondral erosions had strong positive correlations with $R A$ duration $(R=0.62$; $p<0.001)$, the radiologic stage $(R=0.67 ; p<0.001)$ and the blood level of FGF ( $R=0.47$; $p=0.00011)$, weak correlations with HAQ scale $(R=0.22 ; p=0.035), C R P$ $(R=0.24 ; p=0.021)$, DAS28 $(R=0.21$; $p=0.042)$ and the blood level of ACPA ( $R=0.21 ; p=0.04)$. Analysis of correlations between this index and arthroscopic SM indices revealed positive correlations with villous hyperplasia and pannus presence $(R=0.31 ; \quad p=0.03$ and $R=0.52$; $p=0.0001$, respectively). There were also found positive correlations with the following morphological indices: villous hyperplasia with tectorial cell proliferation, angiogenesis and connective tissue disorganization $(R=0.36 ; \quad p=0.02 ; \quad R=0.31 ; \quad p=0.04$ and $R=0.34 ; p=0.03$, respectively).

Multivariate analysis of variance ANOVA/MANOVA carried out to reveal the influence of various joint ultrasound indices on RA activity according to DAS28 parameter (Table 1), showed that sufficient intragroup variance was observed in the indices of exudation into the articular cavity and SM vascularization, which was in line with statistical significance according to Fisher's criterion $(p<0.05)$.

Multivariate ANOVA/MANOVA analysis of the influence of joint ultrasound indices on the radiologic stage in RA patients (Table 2) showed that the most significant intragroup variance was observed in pannus and osteochondral erosion indices, Fisher's criterion was rather high $(p<0.001)$. SM thickness and vascularization had weaker influence on the radiologic stage, but according to Fisher's criterion, the influence was statistically significant $(p<0.01)$.

Discussion. In our investigation ultrasound index of exudation into the articular cavity had definite correlations with the laboratory indices of RA activity: ESR, DAS28, TNF- $\alpha$, which was confirmed by high indices of inflammatory macro- and micro-assessment of SM: inflammatory hyperplasia, SM hyperemia and fibrin presence, SM edema and SM angiogenesis. This proves the data stating that the intensity of exudative inflammatory processes in RA depends on production of pro-inflammatory cytokines, the degree of RA activity and angiogenesis processes [12,13].

Ultrasound index of SM vascularization correlated closely with high laboratory indices of TNF- $\alpha$ and VEGF, ESR and DAS28, which was arthroscopically confirmed by the indices of SM hyperemia and histological indices of SM angiogenesis, lymphoid infiltration. Increase in angiogenesis markers, production of pro-inflammatory cytokines and increase in disease activity underlie the 
enhancement of SM vascularization, cell infiltration and pannus growth processes followed by the development of destructive changes [7, 11, 14].

Ultrasound indices of SM thickness, the presence of pannus and osteochondral erosions correlated closely with the blood levels of FGF in RA patients. According to the literature data [3], FGF activates angiogenesis and fibroblast-like synoviocytes in RA, which, in its turn, promotes pannus growth and joint tissue destruction.

Ultrasound indices of SM thickness, the presence of pannus and osteochondral erosions correlated with the blood levels of ESR and ACPA in RA patients under study, which was proved by a number of investigations showing ESR and ACPA as markers of fast development of destructive changes in RA $[15,16]$.

Ultrasound indices of SM thickness, the presence of pannus and osteochondral erosions correlated with arthroscopic indices of villous hyperplasia and pannus presence as well as morphological indices: villous hyperplasia with tectorial cell proliferation, angiogenesis and connective tissue disorganization.

The established definite correlations between ultrasound indices of SM condition in RA and the indices obtained by macro- and micro-assessment of SM allow us to make a conclusion about the sufficient level of ultrasound SM imaging as compared to arthroscopic and/ or morphological examination, i.e. ultrasound indices correlate with the similar indices of SM macro- and microassessment in all examined RA patients in our study.

Thus, there have been established correlations between the decline in joint ultrasound indices, particularly, exudation into the articular cavity and SM vascularization, and the increase in laboratory indices of immune-inflammatory RA activity (ESR, CRP, DAS28, ACPA, TNF- $\alpha$ ), the rise in the blood levels of angiogenesis markers VEGF, which, in its turn, leads to deterioration of clinical indices (SJC, VAS, HAQ). The decline in ultrasound indices of SM thickness, the presence of pannus and osteochondral erosions correlates closely with increase in RA duration, high levels of aggressive RA course markers (CRP, ACPA) and proliferation factor FGF, which also leads to deterioration of clinical indices (TJC, VAS, HAQ) and progression of joint destruction (the radiologic stage).

Conclusion. Analysis of joint ultrasound indices, such as exudation into the articular cavity and synovial membrane vascularization provides the possibility to evaluate the intensity of angiogenesis and immuneinflammatory processes in rheumatoid arthritis. Ultrasound indices of synovial membrane hypertrophy, pannus formation and osteochondral erosions reflect the severity of proliferative and destructive processes in patients with rheumatoid arthritis. The data obtained provide the possibility of recommending the application of the non-invasive technique of synovial membrane ultrasound for diagnosis as well as monitoring treatment efficacy in patients with rheumatoid arthritis instead of expensive invasive techniques.
Study Funding. This study was carried out in accordance with scientific research plan of Lugansk State Medical University.

Conflicts of interests. There are no conflicts of interest related to the present study.

\section{References}

1. Hitchon C.A., El-Gabalawy H.S. The synovium in rheumatoid arthritis. Open Rheumatol J 2011; 5: 107-114, https://doi.org/10.2174/1874312901105010107.

2. Dhaouadi T., Sfar I., Abelmoula L., Jendoubi-Ayed S., Aouadi H., Ben Abdellah T., Ayed K., Zouari R., Gorgi Y. Role of immune system, apoptosis and angiogenesis in pathogenesis of rheumatoid arthritis and joint destruction, a systematic review. Tunis Med 2007; 85(12): 991-998.

3. Malemud C.J. Growth hormone, VEGF and FGF: involvement in rheumatoid arthritis. Clin Chim Acta 2007; 375(1-2): 10-19, https://doi.org/10.1016/j.cca.2006.06.033.

4. Lyalina V.V., Shekhter A.B. Artroskopiya i morfologiya sinovitov [Arthroscopy and morphology of synovitis]. Moscow: Nauka; 2007; 108 p.

5. Af Klint E., Catrina A.I., Matt P., Neregråd P., Lampa J., Ulfgren A.K., Klareskog L., Lindblad S. Evaluation of arthroscopy and macroscopic scoring. Arthritis Res Ther 2009; 11(3): R81, https://doi.org/10.1186/ar2714.

6. Ten Cate D.F., Luime J.J., Swen N., Gerards A.H., De Jager M.H., Basoski N.M., Hazes J.M., Haagsma C.J., Jacobs J.W. Role of ultrasonography in diagnosing early rheumatoid arthritis and remission of rheumatoid arthritis a systematic review of the literature. Arthritis Res Ther 2013; 15(1): R4, https://doi.org/10.1186/ar4132.

7. Fukae J., Tanimura K., Atsumi T., Koike T. Sonographic synovial vascularity of synovitis in rheumatoid arthritis. Rheumatology (Oxford) 2014; 53(4): 586-591, https://doi. org/10.1093/rheumatology/ket311.

8. Korobkova A.S., Agafonov A.V., Tuev A.V., Vasilets L.M., Khlynova O.V., Zhelobov V.G., Grigoriadi N.E. Possibilities of ultrasonography in assessment local inflammation in rheumatoid arthritis. Modern Problems of Science and Education 2013; 6. URL: http://www.science-education.ru/ru/ article/view?id=11383.

9. Kurzantseva O.M., Murashkovskiy A.L., Trofimov A.F., Fedorov V.I. Ultrasound differential diagnosis of deforming osteoarthrosis and rheumatoid arthritis in knee joint lesion. SonoAce International 2005; 13: 78-81. URL: http://www. medison.ru/si/art198.htm.

10. Ozgocmen S., Ozdemir H., Kiris A., Bozgeyik Z., Ardicoglu O. Clinical evaluation and power Doppler sonography in rheumatoid arthritis: evidence for ongoing synovial inflammation in clinical remission. South Med J 2008; 101(3): 240-245, https://doi.org/10.1097/SMJ. 0b013e318164e16a.

11. Hama M., Uehara T., Takase K., Ihata A., Ueda A., Takeno M., Shizukuishi K., Tateishi U., Ishigatsubo Y. Power Doppler ultrasonography is useful for assessing disease activity and predicting joint destruction in rheumatoid arthritis patients receiving tocilizumab - preliminary data. Rheumatol Int 2012; 32(5): 1327-1333, https://doi.org/10.1007/s00296011-1802-5.

12. Zufferey P., Möller B., Brulhart L., Tamborrini G., Scherer A., Finckh A., Ziswiler H.R. Persistence of ultrasound 
synovitis in patients with rheumatoid arthritis fulfilling the DAS28 and/or the new ACR/EULAR RA remission definitions: results of an observational cohort study. Joint Bone Spine 2014; 81(5): 426-432, https://doi.org/10.1016/j.jbspin.2014.04.014.

13. Ramírez J., Ruíz-Esquide V., Pomés I., Celis R., Cuervo A., Hernández M.V., Pomés J., Pablos J.L., Sanmartí R., Cañete J.D. Patients with rheumatoid arthritis in clinical remission and ultrasound-defined active synovitis exhibit higher disease activity and increased serum levels of angiogenic biomarkers. Arthritis Res Ther 2014; 16(1): R5, https://doi.org/10.1186/ar4431.

14. Dougados M., Devauchelle-Pensec V., Ferlet J.F., Jousse-Joulin S., D'Agostino M.A., Backhaus M., Bentin J., Chalès G., Chary-Valckenaere I., Conaghan P., Wakefield R.J., Etchepare F., Gaudin P., Grassi W., van der Heijde D.,
Mariette X., Naredo E., Szkudlarek M. The ability of synovitis to predict structural damage in rheumatoid arthritis: a comparative study between clinical examination and ultrasound. Ann Rheum Dis 2013; 72(5): 665-671, https://doi.org/10.1136/ annrheumdis-2012-201469.

15. Rönnelid J., Wick M.C., Lampa J., Lindblad S., Nordmark B., Klareskog L., van Vollenhoven R.F. Longitudinal analysis of citrullinated protein/peptide antibodies (anti-CP) during 5 year follow up in early rheumatoid arthritis: anti-CP status predicts worse disease activity and greater radiological progression. Ann Rheum Dis 2005; 64(12): 1744-1749.

16. Valesini G., Alessandri C. Anticitrullinated protein/ peptide antibodies and rheumatoid factors: two distinct autoantibody systems. Arthritis Res Ther 2009; 11(5): 125, https://doi.org/10.1186/ar2786. 